\title{
Enhanced Magnetoelectric Voltage in Ferrite/PZT/Ferrite Composite for AC Current Sensor Application
}

DOI: https://doi.org/10.1007/s10854-018-9576-0

\author{
A. Aubert · V. Loyau • G. Chaplier . \\ F. Mazaleyrat • M. LoBue
}

July 2018

\begin{abstract}
In this paper, we report the fabrication of a rare-earth free current sensor based on a PZT/NiCoZn-ferrite magnetoelectric (ME) trilayer composite disk. To improve the sensitivity of the sensor, the structure uses an in-plane series connection, which increases the ME voltage by two for a fixed volume. Then, we propose a full characterization of the sensor : electrical modeling, low and high frequency limits, sensitivity, linearity, distortion and resolution. The device is also evaluated under sinus, square, and triangle waveform currents, which are excitation signals commonly used in the field of electrical engineering. The current sensor shows high current sensitivity $(90 \mathrm{mV} / \mathrm{A})$, good linearity from $0.001 \mathrm{~A}$ to $30 \mathrm{~A}$ and low added impedance $(0.01 \Omega)$ in a frequency range of $10 \mathrm{~Hz}-30 \mathrm{kHz}$ for a very compact structure $\left(4 \mathrm{~cm}^{3}\right)$. It also exhibits a high resolution of $1 \mathrm{~mA}$ (for a 1 A peak signal) and good stability.
\end{abstract}

Keywords Magnetoelectric composite · Current sensor · Ferrite · PZT . Spark Plasma Sintering · Rare-earth free

A. Aubert, corresponding author

SATIE UMR 8029 CNRS, ENS Paris-Saclay, Université Paris-Saclay, 61 Avenue du Prsident Wilson, 94235 Cachan, France

E-mail: alex.aubert@satie.ens-cachan.fr

V. Loyau · G. Chaplier · F. Mazaleyrat · M. LoBue

SATIE UMR 8029 CNRS, ENS Paris-Saclay, Université Paris-Saclay, 61 Avenue du Prsident Wilson, 94235 Cachan, France 


\section{Introduction}

The magnetoelectric (ME) effect is defined as the variation of the electric polarization induced by an applied magnetic field. In magnetoelectric laminated composites, the magnetoelectric effect is due to the magnetic-mechanical-electric transform, induced by the mechanical coupling between a magnetostrictive and a piezoelectric layer. To enable the piezomagnetic behavior of the magnetostrictive material, the magnetic layer needs to be polarized by a DC magnetic field with a superimposed AC field [1].

Recently, the progress made in the field of magnetoelectric (ME) laminate composites allows potential high performance applications such as magnetic field sensors [2-5], current sensors [6-16], gyrators [17], filters [18] and more recently, memory devices [19-23]. In the field of current sensors, magnetoelectric composites show many advantages : (i) high performances, (ii) low cost, (iii) power supply free, (iv) galvanic isolation and (v) simplified electronic circuits [24].

Different types of magnetoelectric AC current sensors have been reported in the literature. In case of current sensor for electronic device applications, the main goal is to obtain high performances sensors (sensitivity, linearity, resolution) with large frequency bandwidth. This was achieved in ring-type sensor [6,7], slice-type sensor [11] and packaged current sensor [8,9]. However, all these sensors use rare-earth elements as magnetostrictive materials which are either Terfenol-D or Samfenol. Currently, one of the biggest challenge in magnetic materials is to find reliable substitutes to rare-earth elements, because of their potential supply risk [25]. Recently, we have demonstrated that NiCoZn-ferrite (NCZF) has great potential to replace Terfenol-D for magnetoelectric purpose $[12,13,26]$.

In this study, a current sensor based on a NCZF/PZT/NCZF trilayer composite disk is proposed. This structure was chosen because of its ease of fabrication and good magnetoelectric performances in spite of being rare-earth free [26]. Moreover, the magnetoelectric composite is here modified in order to use the in-plane series connected structure (see in Fig. 1). This connection was recently used by Fang et al. in Metglas/Mn-doped PMNT fibers laminate composite to reduce the magnetic noise in magnetoelectric magnetic field sensors [2]. They also reported a significant enhancement of the ME voltage with such structure. In this work, the in-plane series connection is used to enhance the ME voltage of the composite, which permits to increase the sensitivity of the sensor for an unaltered volume. Here, the magnetic excitation field (image of the current to be measured) is produced by a coil connected in series with the measured signal. Due to the high sensitivity of our composite, this coil is composed of a unique turn. Hence, the perturbation induced by the sensor in the measured current is expected to be of little influence, because of the low impedance added by a one-turn coil. On the other hand, this setup results in a capacitance $C_{0}$ of the ME composite four times lower (surface divided by two and two capacitance connected in series), which extend the low frequency limit because ME composite acts like high-pass filter in quasi-static mode [27]. The final structure of the sensor is obtained by placing the ME composite between two flat permanent magnets in parallel configuration, which produce the magnetic polarizing field. Strontium hexaferrites magnets were chosen because of their many advantages : (i) low cost, (ii) ease of fabrication, (iii) high resistivity (no eddy-currents), (iv) ease of polarization, and (v) rare-earth 
free. The sensor is then tested under three different electric signals (sinus, square and triangles) to fully explore its potential.

The paper is organized as follows. In Sec. II, the fabrication process of the ME composite is presented, as well as the sensor manufacturing. In Sec. III, the benefit of using in-plane series connection is highlighted compared with basic trilayer composites. Also, the magnetic field homogeneity produced by the two magnets in the ME composite area is verified by numerical calculation. Then, the sensor is fully characterized : electrical modeling, sensitivity, low and high frequency limit, linearity, distortion and resolution. Finally, the sensor presented in this study is compared with the ones previously reported in the literature.

(a) Basic Connection

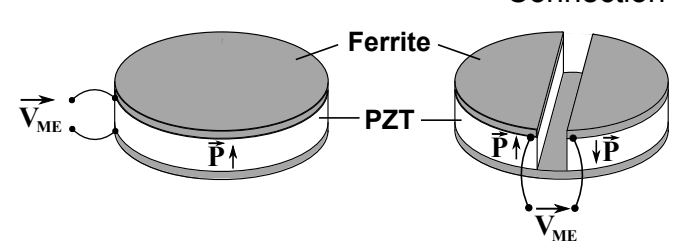

(b) In-Plane Serie

Connection

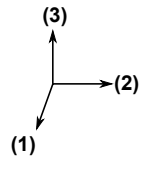

Fig. 1 (a) Basic trilayer ME composite and (b) ME composite with in-plane series connected structure.

\section{Experimental methods}

2.1 Fabrication of the magnetoelectric composite

Nano-sized grains ferrite disks of composition $\left(\mathrm{Ni}_{0.973} \mathrm{Co}_{0.027}\right)_{0.875} \mathrm{Zn}_{0.125} \mathrm{O}_{4}(\mathrm{NCZF})$ were produced by a reactive-sintering using Spark Plasma Sintering (SPS) method. This method allows to obtain high dense ceramic pellets with nanosized grains in a few minutes $[28,29]$. The process was described in details elsewhere [26]. The obtained disks (diameter: $10 \mathrm{~mm}$, thickness: $2 \mathrm{~mm}$ ) were fully re-oxidized by an annealing at $1000{ }^{\circ} \mathrm{C}$ for $1 \mathrm{~h}$ in air. By means of silicon carbide paper, the thickness of the disks were reduced to $0.25 \mathrm{~mm}$ each. The PZT disks have been purchased (PZ27 from Ferroperm, thickness: $1.3 \mathrm{~mm}$, diameter: $10 \mathrm{~mm}$ ). To make basic trilayer structure, the PZT is pasted between the two ferrite disks using silver epoxy (Epoteck E4110), and the final trilayer is sketched Fig. 1 (a).

To make a structure with in-plane series connection, one needs to cut along the diameter direction one ferrite and one PZT disks. This was done using a diamond saw (Strueurs Secotom), getting two halves of a ferrite and PZT disks. The two PZT half disks were pasted on a complete ferrite disk and mounted in antiparallel polarization direction with series connection. Lastly, the two half ferrite disks were pasted with the conductive epoxy on the two PZT half disks. Figure 1 (b) illustrates the structure of the ME composite. The groove that separates the two PZT parts was filled by insulating epoxy to enhance the mechanical strength. The differential ME voltage is measured between the two top electrodes of the two half PZT disks. 


\subsection{Fabrication of the sensor}

Due to the high permeability and the low demagnetizing field of thin soft ferrites, it has been shown that these materials require low optimum DC magnetic fields to polarize the ME composite [26]. The low DC field working point can be achieved by different magnet setups. Among them, we have chosen a structure in which the ME composite is inserted in the gap between two flat magnets in a parallel configuration (see in Fig. 2). Commercial strontium ferrites (textured but nonpolarized) were cut in parallelepiped shape $\left(18 \times 18 \times 2.5 \mathrm{~mm}^{3}\right)$ and polarized under a DC field to reach the optimum bias DC field.

The ME composite was then inserted in a one turn coil made with a copper strip (10 mm width, $0.1 \mathrm{~mm}$ thick) wounded around a plastic sleeve (see in Fig. 2). The produced AC magnetic field has its main component in the direction (1) of the ME composite (see in Fig. 1) and the coil is roughly as long as it is wide $\left(\sim 10 \times 14 \mathrm{~mm}^{2}\right)$. The coil produces a magnetic field of $0.1 \mathrm{mT}$ when a current of $1 \mathrm{~A}$ is injected. The ME composite with its coil is placed in the gap between the two permanent magnets allowing a very compact structure $\left(4 \mathrm{~cm}^{3}\right)$. A photograph of the final prototype is given in the inset Figure 2.

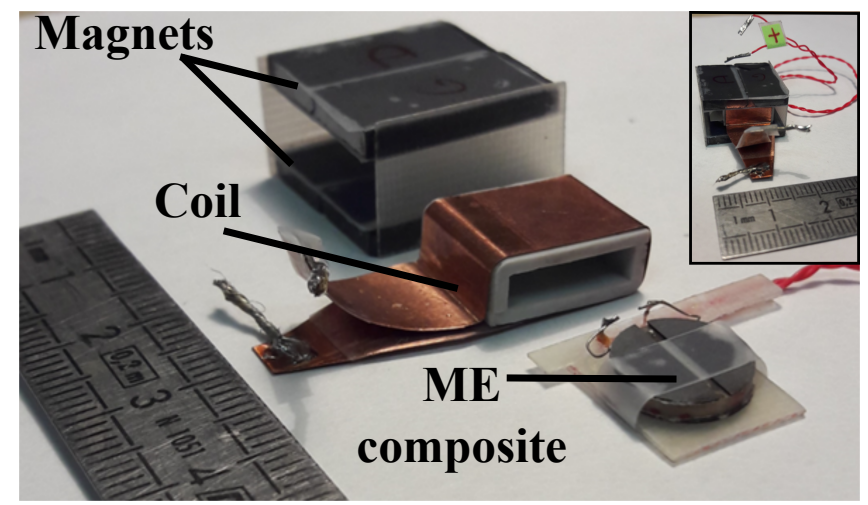

Fig. 2 Photograph of the three different components of the current sensor before assembly and after assembly in the inset.

\subsection{Measurement procedures}

To characterize the magnetoelectric composite, the magnetoelectric coefficient is measured as function of a continuous magnetic field $H_{D C}$ produced by an electromagnet applied in the direction (1) of the trilayer magnetoelectric sample. A small external AC field is superimposed in the same direction $(1 \mathrm{mT}, 1 \mathrm{kHz})$ produced by Helmoltz coils (see Fig. 1 (b)). The magnetoelectric voltage is then measured with a lock-in amplifier (EG\&G Princeton Applied Research Model 5210) having a high input impedance of $100 \mathrm{M} \Omega$.

Regarding the sensor, electrical characterization is made using a Keysight E4980A Precision LCR Meter. To characterize the sensor at high frequency, the 
Table 1 Characteristics of the reference sensor TPC312 (probe) w/ TCPA300 (amplifier).

\begin{tabular}{lllll}
\hline Bandwidth & $\begin{array}{l}\text { Rise } \\
\text { Time }\end{array}$ & $\begin{array}{l}\text { Low and High } \\
\text { Current Sens. }\end{array}$ & $\begin{array}{l}\text { Max. RMS } \\
\text { Current }\end{array}$ & Accuracy \\
\hline $\mathrm{DC}-100 \mathrm{MHz}$ & $\leq 3.5 \mathrm{~ns}$ & 1 and $10 \mathrm{~A} / \mathrm{V}$ & 3.5 and $21.2 \mathrm{~A}$ & $\pm 1 \%$ \\
\hline
\end{tabular}

current to be measured is provided by a power amplifier (NF-HAS-4181) loaded by a $4 \Omega$ power resistor. For high magnitudes of current, the signal measured by the sensor is provided by a power amplifier Samson (SX1800) loaded by a $4 \Omega$ power resistor. A commercial active current probe (Tektronix TCP312) is used with an amplifier (TCPA300) as reference for the current measurement. The main characteristics of this sensor are provided in Table 1 to justify its use as a reference. To characterize the sensor, the magnetoelectric voltage is measured by a passive voltage probe (input impedance : $10 \mathrm{M} \Omega$ ) connected to an oscilloscope. The linearity was characterized by measuring the voltage using the lock-in amplifier. The resolution of the sensor is measured using a HP 34401A Multimeter (6 digits) while the signal is induced by a waveform generator Agilent 33250A.

\section{Results \& Discussion}

\subsection{Characterization of the magnetoelectric composites}

In Figure 3 is plotted the ME voltage measured as function of $H_{D C}$ for both the basic and the in-plane series connected structure trilayer. The maximum ME voltage is $0.42 \mathrm{~V}$ for the basic composites while it is increased almost by two for the in-plane series structure $(0.78 \mathrm{~V})$. This gives a sensitivity of $0.78 \mathrm{~V} / \mathrm{mT}$ to our composite. Moreover, the optimum DC magnetic field $H_{\max }$ required to reach the maximum ME voltage is reduced to $8500 \mathrm{~A} / \mathrm{m}(\sim 11 \mathrm{mT})$. The corresponding transverse magnetoelectric coefficient $\alpha_{31}$ is calculated to be $567 \mathrm{mV} / \mathrm{A}$ when normalized on the total thickness of the ME sample $(1.8 \mathrm{~mm})$.

The maximum ME voltages (at $H_{D C}=8500 \mathrm{~A} / \mathrm{m}$ ) were measured for different directions of the magnetic fields applied in the $(1,2)$ plane of the sample (see inset Fig. 4). The AC and DC magnetic fields direction is fixed in the air gap of the electromagnet while the sample rotates by angles $\theta$ around the axis (3). Figure 4 shows a large dependence on $\theta$ of the ME voltage, mainly due to the demagnetizing effect produced by the air gap between the two half ferrite discs on the top of the ME composite. In fact, magnetic fields applied perpendicularly to the air gap increase the demagnetizing effect, resulting in a decrease of the internal magnetic field, and consequently in a weakening of the ME effect. The ME composite shows a relatively strong directivity in the $(1,2)$ plane. The dependence on the angle should have low impact on the sensor's use because the composite will be oriented in its optimal position $(\theta=0)$ and is not supposed to move afterwards. Moreover, this configuration should decrease the sensitivity to perpendicular magnetic field induced by others devices, which is beneficial for our sensor. 


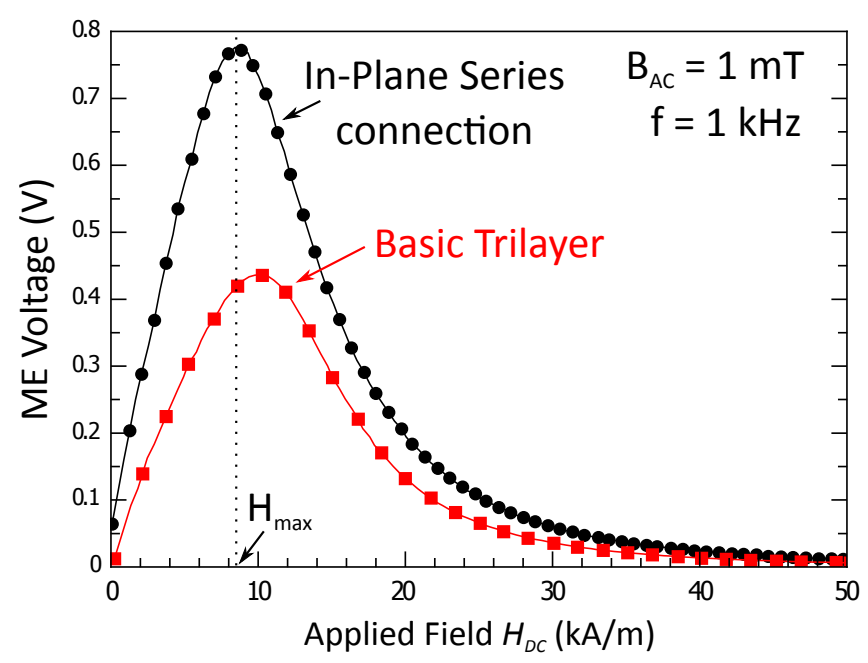

Fig. $3 \mathrm{ME}$ voltage produced in the trilayer for basic composite (in red) and for the ME composite with in-plane series connection (in black) as function of the applied field.

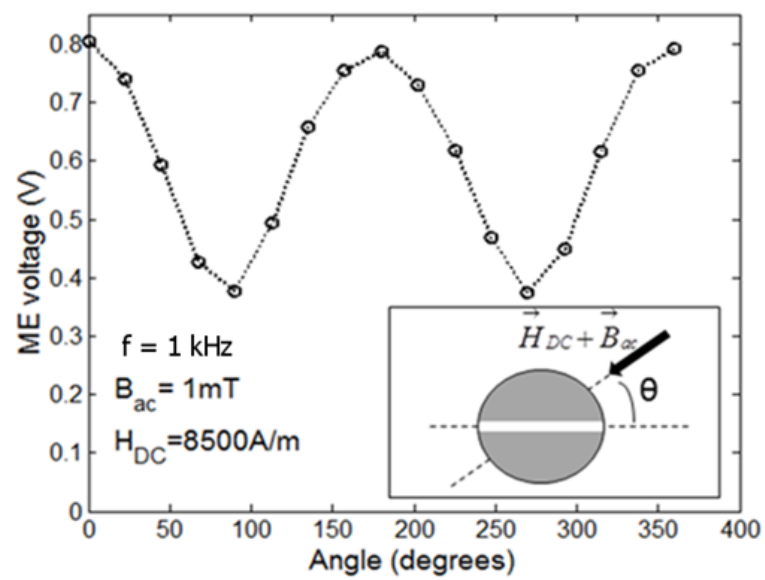

Fig. 4 Maximum ME voltage as function of the applied AC and DC magnetic field direction. The inset indicates the direction of the applied fields in a top view.

\subsection{Homogeneity of the polarizing field for the sensor}

As mentioned before, ME sensors might be attractive because of their compact structure which implies to reduce the space between the magnets that produces the DC field. However, without a proper managing of the device geometry, the magnetic field crossing the ME composite can be spatially inhomogeneous, hence compromising ME performances. To get an homogeneous field of the required intensity $(\sim 11 \mathrm{mT})$ in the area of the ME trilayer, we performed numerical calculations using Finite Element Method (FEM) software (ANSYS Maxwell). The 
results are presented in Figure 5. It appears that the differences in field never exceed $1.8 \mathrm{mT}$, hence validating the geometrical structure of the magnets.

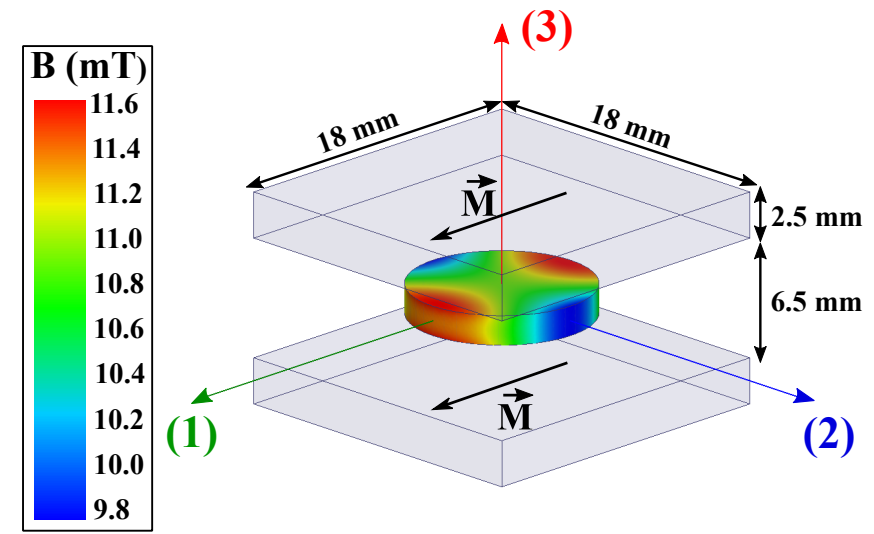

Fig. 5 Numerical calculation (FEM) of the DC magnetic field produced by two permanent magnet (Sr hexaferrite) in the area of the ME composite. The two black arrows indicate the direction of the remanent magnetization in the magnets.

3.3 Electrical modeling of the sensor

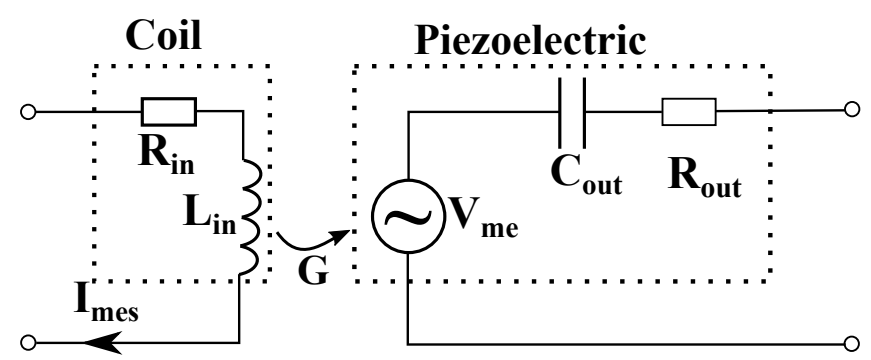

Fig. 6 Equivalent electrical modeling of the sensor at low frequency including the one-turn coil (left) and the magnetoelectric composite (right) [27].

The input of our current sensor is a one-turn coil, which can be electrically modeled by a resistance connected in series with an inductance (see left part of Fig. 6). Here, the measured current is connected in series to our sensor, which can hence disrupt the measured signal because of the adding impedance. Thus, the coil needs to be optimized (size and number of turns) to ensure an impedance as low as possible in order to minimize the disturbances in the measured signal. The input serial inductance $\left(L_{i n}\right)$ and resistance $\left(R_{i n}\right)$ of the coil are measured to be $200 \mathrm{nH}$ and $0.01 \Omega$, respectively. The general impedance of the one-turn coil $\left(Z_{i n}=R_{i n}+j \omega L_{i n}\right)$ is then plotted as function of the frequency from $10 \mathrm{~Hz}$ to $30 \mathrm{kHz}$ in Figure 7 (black line). The impedance remains almost constant within 
this range of frequency. It is known that the number of turns in a coil influences its general impedance. To highlight this effect, the impedance of a 10 turns coil is also plotted (red line). This coil exhibits the same active surface than the previous oneturn coil presented, but was divided into 10 turns, hence multiplying the resistance and inductance of the coil by a factor $\mathrm{N}^{2}\left(L_{i n} \sim 20 \mu \mathrm{H}, R_{\text {in }} \sim 1 \Omega\right)$. In this case, the impedance is increased by a factor $100\left(Z_{i n} \sim 1 \Omega\right)$ compared to the one-turn coil. This results points out the importance of minimizing the number of turns of the input coil to ensure low disturbances of the measured signal. Of course, reducing the number of turns will decrease the sensitivity of the sensor. However, in our case, the high ME voltage produced by the ME composite NCZF/PZT/NCZT allows us to use a one-turn coil. Using a one turn coil should also be beneficial to decrease the joule heating lost at currents greater than $5 \mathrm{~A}$.

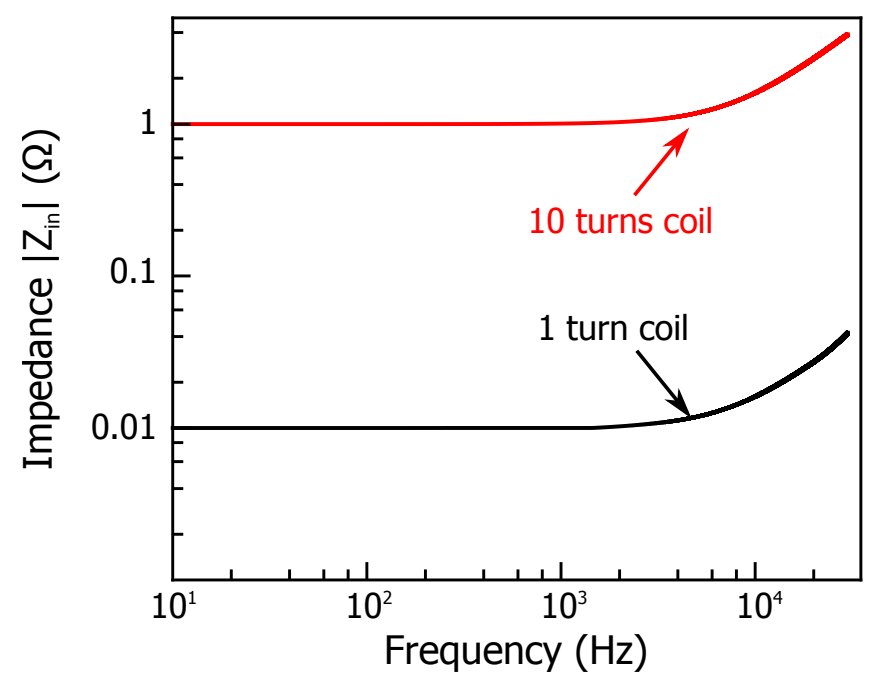

Fig. 7 Impedance $\left|Z_{i n}\right|$ of a coil made of 1 turn (black) or 10 turns (red) as function of frequency. The resistance of the coil in both case is $0.01 \Omega$.

For electrical engineering applications, the input impedance of the one-turn coil $(0.01 \Omega)$ is assumed to be low enough to induce negligible perturbation in the measured signal. Nevertheless, this impedance can be diminished by increasing the thickness of copper foil, hence reducing the resistance value by a factor 10 or more. Moreover, increasing the thickness will allow higher current flows, which would be advantageous for high current sensing.

The output of our current sensor is a piezoelectric layer. According to Texas Instruments, piezoelectric can be electrically modeled by a voltage source with a series capacitor and resistor at low frequency (see right part of Fig. 6) [27]. The piezoelectric loaded with a resistive charge can hence be seen as a high-pass filter of first order. The output capacitance $\left(C_{\text {out }}\right)$ and resistance $\left(R_{\text {out }}\right)$ are measured to be $197 \mathrm{pF}$ and $16 \mathrm{k} \Omega$, respectively. This impedance will mainly influence the low frequency limit of the sensor (see Sec. 3.4). 

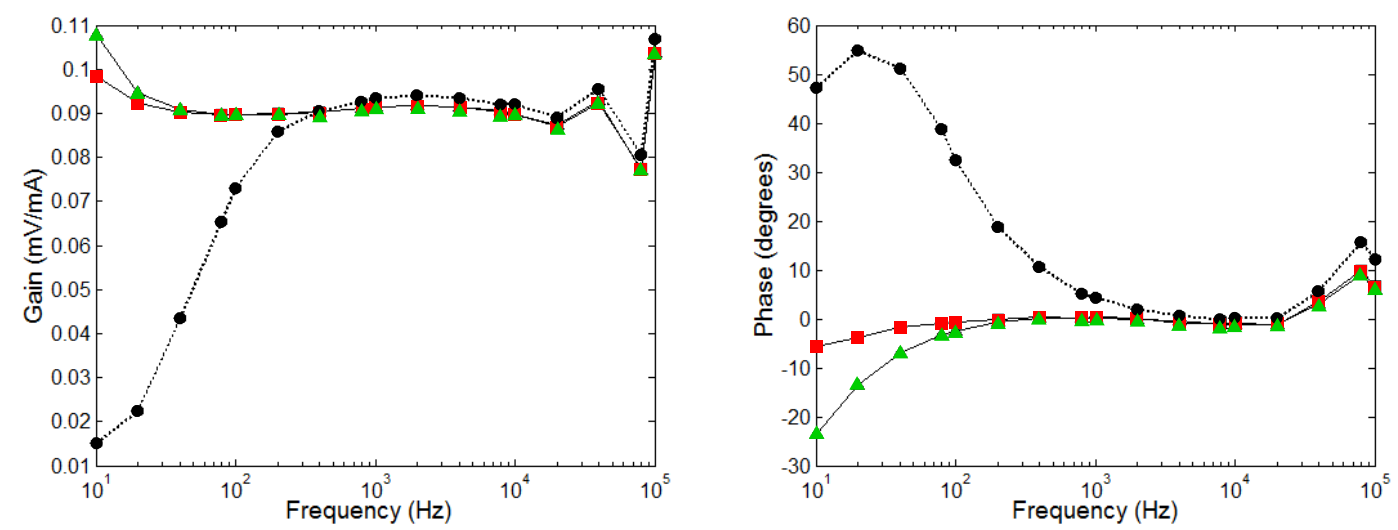

Fig. 8 (a) Gain $(\mathrm{mV} / \mathrm{mA})$ and (b) Phase (degrees) of the ME current sensor measured for frequency between $10 \mathrm{~Hz}$ and $100 \mathrm{kHz}$. Black circle symbols: output of the ME current sensor connected to a $10 \mathrm{M} \Omega$ passive voltage probe. Green triangle symbols: output of the ME sensor connected to the buffer circuit. Red square symbols: output of the ME sensor connected to the buffer circuit with a $200 \mathrm{M} \Omega$ resistor connected in parallel at its input.

\subsection{Low frequency limit of the sensor}

The gain (amplitude and phase) of the ME current sensor was measured in a frequency range spanning from $10 \mathrm{~Hz}$ to $100 \mathrm{kHz}$ with a $1 \mathrm{~A}$ RMS current. The results are given in Figure 8 (black circle symbols). As expected from electrical modeling, the gain decreases at low frequency $(<200 \mathrm{~Hz})$. In fact, the experimental low cut-off frequency is around $80 \mathrm{~Hz}$. This frequency is also determined theoretically by the ouput impedance of the sensor, given by the following expression : $f_{c}=1 /\left(2 \pi C_{\text {out }} R_{\text {tot }}\right)$, where $R_{\text {tot }}$ corresponds to the output resistance of the sensor $\left(R_{\text {out }}\right)$ connected in series with the input impedance of the voltage probe $(10 \mathrm{M} \Omega)$. This calculation gives a low cut-off frequency of $80.66 \mathrm{~Hz}$ which is in good agreement with experimental data, hence validating the electrical modeling at low frequency (Fig. 6).

To bypass this low frequency limit, a high impedance buffer circuit can be added to increase the output impedance of the sensor, hence decreasing the low cut-off frequency. The buffer circuit is sketched in Figure 9. It is based on a unitgain circuit made with a low-cost (J-Fet input) operational amplifier (TL081). The low input bias current in the non-inverter input is supplied by a bootstrap circuit made by the diodes D1, D2, and the resistors R2, R3. The voltage at the two diodes is much lower than their threshold voltage, and consequently, their equivalent resistance is high. This structure permits to reach an input resistance as high as $100 \mathrm{G} \Omega$. When the high impedance buffer circuit is connected to the current sensor, the gain at low frequency measured by the passive voltage probe remains above $0.09 \mathrm{~V} / \mathrm{A}$ and the phase shift is limited (green triangle symbols in Fig. 8). The slight rise in the gain around $10 \mathrm{~Hz}$ is probably due to the gain instability of the buffer circuit at low frequency. This effect is weakened when a $200 \mathrm{M} \Omega$ resistor is connected at the input of the buffer circuit in parallel (red square symbols in Figure 8). Hence, at low frequency, the gain is between 0.09 and $0.1 \mathrm{~V} / \mathrm{A}$ and the phase shift never exceeds 7 degrees. 


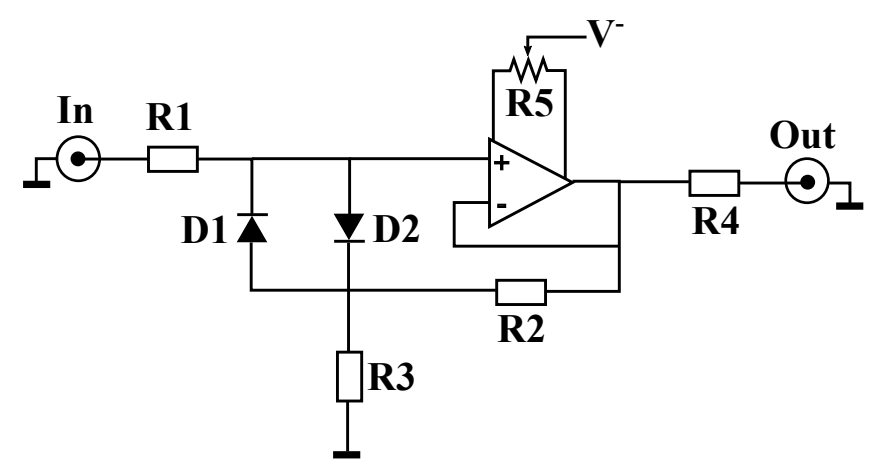

Fig. 9 Electrical scheme of the high input impedance buffer circuit. $R 1=R 2=100 \mathrm{M} \Omega R 3=$ $10 \mathrm{k} \Omega, R 4=51 \Omega, R 5=20 \mathrm{k} \Omega$

\subsection{High frequency limit of the sensor}

At high frequency, the upper limit of the current sensor should be determined by the resonance frequencies of the ME composite. From Figure 8, it seems that the gain becomes non-constant from approximately $30 \mathrm{kHz}$.

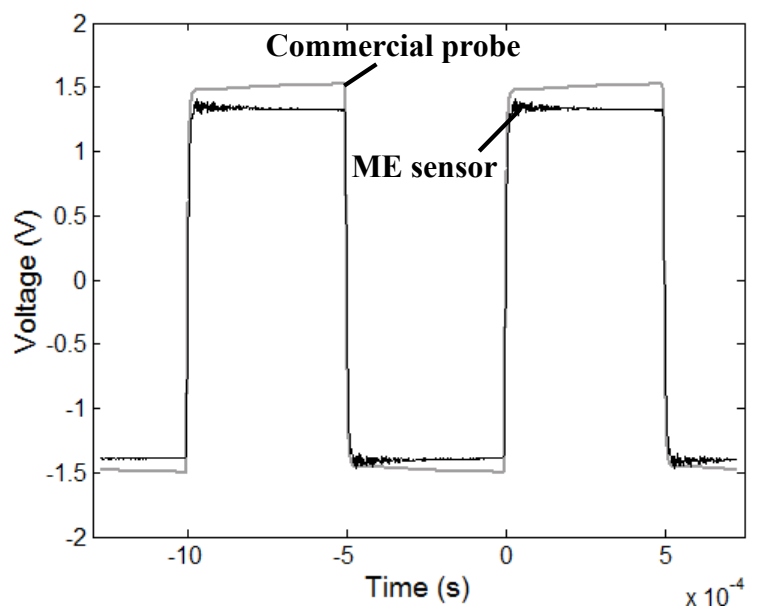

Fig. 10 Measurement of a square waveform current of $1.5 \mathrm{~A}$ pk at $1 \mathrm{kHz}$. The voltage of the ME sensor is increased 10 times for better comparison.

This upper limit can be highlighted when the current sensor is subjected to a fast variation of current, because the resonance oscillations appear in the ME voltage. Thus, this effect can be illustrated by applying a square waveform signal $(1 \mathrm{kHz}, 1.5 \mathrm{~A} \mathrm{pk})$ as shown in Figure 10. The current measured by a commercial probe is also plotted for comparison. The gain and phase between the reference signal and the measured one are in agreement. However, just after the rising edge, the ME voltage shows high frequency oscillations. This effect is zoomed in Figure 11. These oscillations can be regarded as noise and the Signal to Noise 
Ratio (SNR) was calculated to be $40 \mathrm{~dB}$. To analyze them, the Fast Fourier Transform (FFT) of the ME voltage overshoots was calculated and the results are given in the inset in Figure 11. Six main frequencies are present in the overshoot signal spectrum.

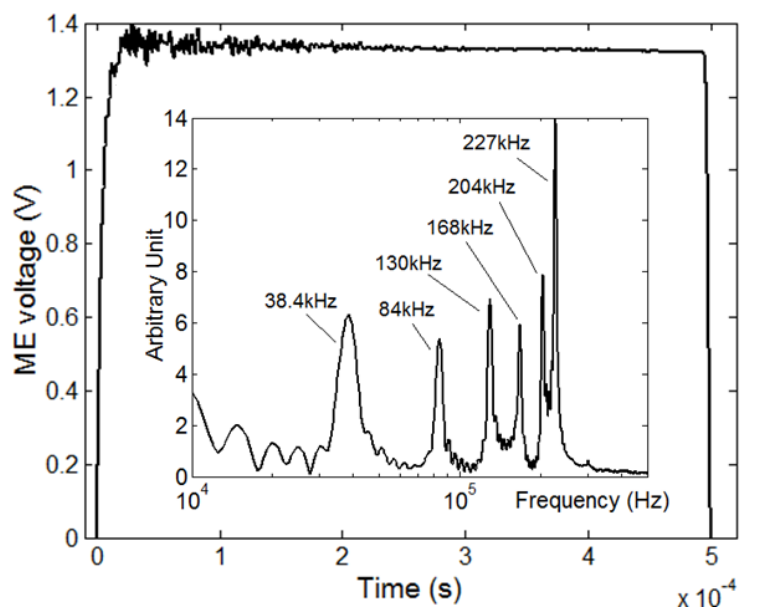

Fig. 11 Focus on the voltage overshoot produced by a square waveform current and its calculated Fast Fourier Transform (FFT) in the inset.

To confirm these frequencies are caused by the resonance phenomenon, the evolution of the ME gain was measured up to $500 \mathrm{kHz}$, applying a sine waveform current to the sensor (see Figure 12). Usually, for a ME disk of $10 \mathrm{~mm}$ diameter, the main resonant peak arises around $300 \mathrm{kHz}[30,31]$. Here it appears at $228 \mathrm{kHz}$, probably because of the large volume fraction of PZT compared to ferrite and because of the cutting of the disks. The peaks at $85 \mathrm{kHz}, 130 \mathrm{kHz}, 167 \mathrm{kHz}$, $204 \mathrm{kHz}$, and $228 \mathrm{kHz}$ correspond to the frequencies retrieved by the FFT for the $\mathrm{ME}$ voltage measuring a square signal. These frequencies should also be due to the geometry of the ME composite, which is not a perfect disk. This confirms the impact of the electro-mechanical resonance on oscillations induced by the voltage overshoot. However, the peak at $38.4 \mathrm{kHz}$ was not identified and its origin remains unknown.

Nevertheless, these oscillations should not be an issue when measuring currents in power electronic devices (e.g. static DC-DC converters or power inverters). Indeed, such circuits are generally enough inductive to have smooth variation of current, resulting in triangle waveform signal. The ME voltage measured for a triangle waveform current $(1.5 \mathrm{~A} \mathrm{pk}, 1 \mathrm{kHz})$ is plotted in Figure 13. The ME current sensor exhibits good linearity without phase shift or oscillation noise.

\subsection{Linearity and distortion of the sensor}

In order to study the linearity of the sensor, a sine waveform current $(1 \mathrm{kHz})$ with amplitudes varying from 0.001 to $30 \mathrm{~A}$ (RMS) has been applied. The results 


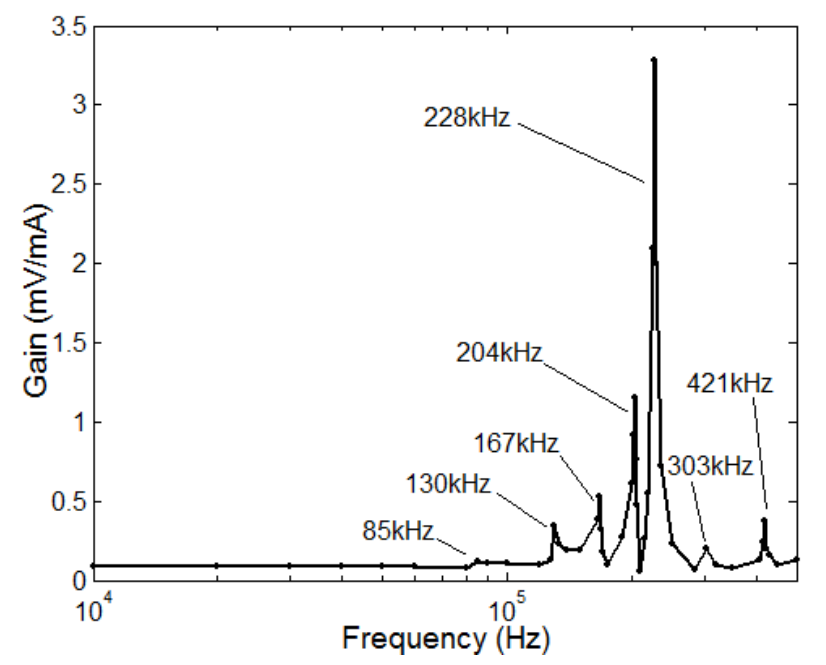

Fig. 12 Gain of the ME current sensor measured as function of the frequency from $10 \mathrm{kHz}$ to $500 \mathrm{kHz}$.

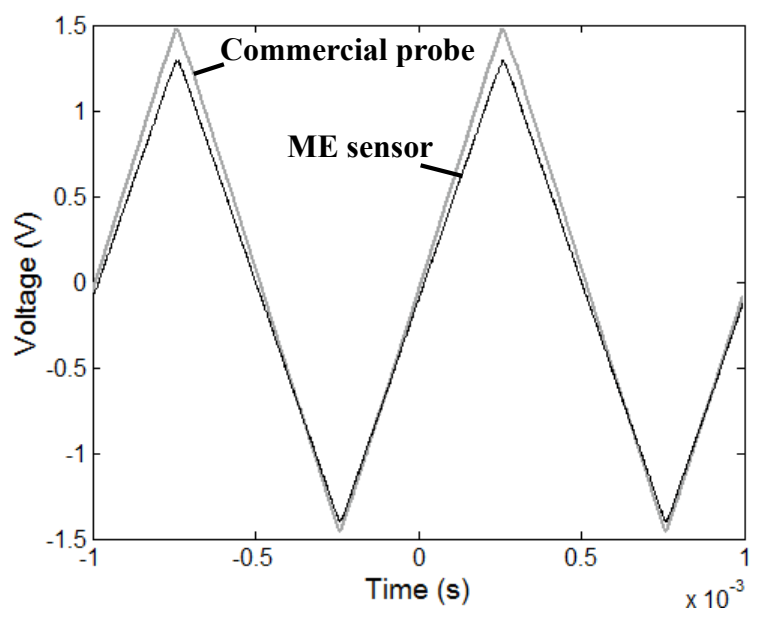

Fig. 13 Measurement of a triangle waveform current of $1.5 \mathrm{~A}$ pk at $1 \mathrm{kHz}$. The voltage of the ME sensor is increased 10 times for better comparison.

are given in Figure 14 (a). In the inset, we plotted the calculated coefficient of determination $R^{2}$. This coefficient was calculated to be 0.999971 , showing the good linearity of our sensor for this range of current.

Then, harmonic distortion due to the amplitude of the current was measured [3, 32-35]. The corresponding ME voltages were recorded by a digital oscilloscope (without passing through the buffer), and the FFT was calculated. The results of the harmonic measurements are presented in Figure 14 (b). The amplitudes of the second $(2 \mathrm{kHz})$, third $(3 \mathrm{kHz})$, and fourth $(4 \mathrm{kHz})$ harmonics are at least $40 \mathrm{~dB}$ lower than the fundamental. Considering the three harmonics, the Total Harmonic 
Distorsion $\left(\mathrm{THD}_{F}\right)$ is calculated to be less than $0.02 \%$. This validates the low harmonic distortion of the sensor for this range of current.
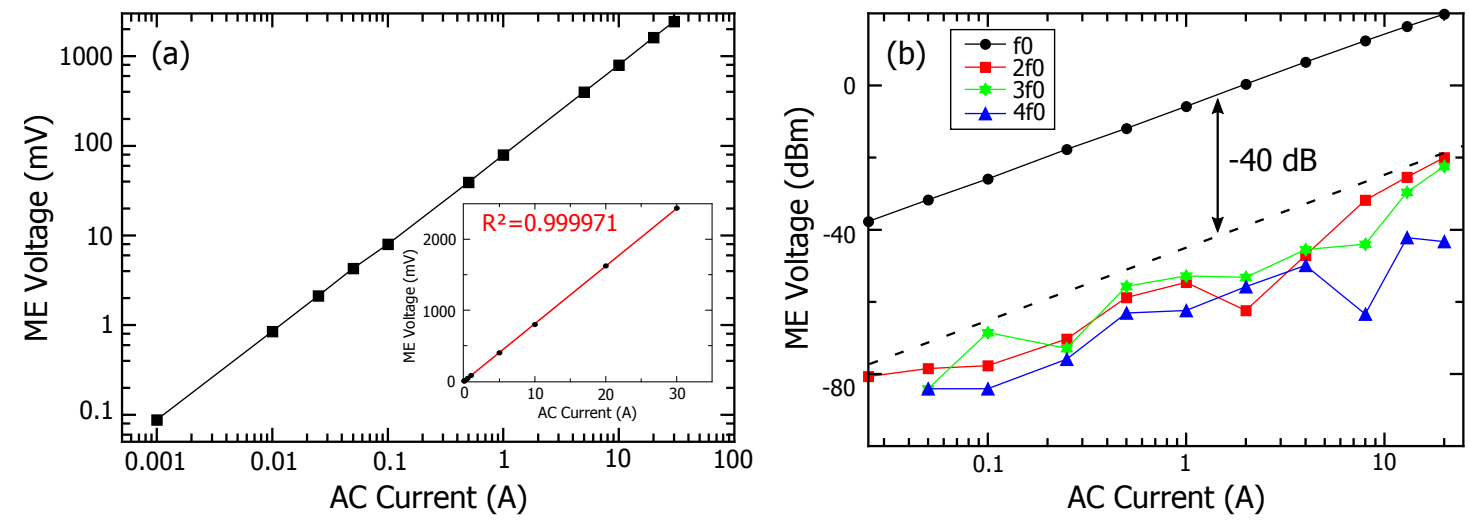

Fig. 14 (a) ME Voltage as function of AC current at $1 \mathrm{kHz}$ (RMS values) and the linearity for the same range of current is plotted in the inset (b) Fundamental (circle), second harmonic (square), third harmonic (star) and fourth harmonic (triangle) of the ME voltage (in $\mathrm{dBm}$ ) as a function of the amplitude of a sinusoidal AC current at $1 \mathrm{kHz}$.

\subsection{Resolution of the sensor}

To evaluate the resolution of the sensor, the ME voltage is measured for small AC current variations. The excitation signal is a $1 \mathrm{~A}$ peak sinusoidal current under the frequency of $5 \mathrm{kHz}$, and the variation in the current's magnitude is of $0.001 \mathrm{~A}$. The measured current starts at $1.000 \mathrm{~A} \mathrm{pk}$ and is incremented five times every 30 seconds until reaching $1.005 \mathrm{~A}$ pk and is then decremented back to $1.000 \mathrm{~A}$ pk. The ME voltage measured as function of time is plotted in Figure 15. It appears that the input signal change $\delta \mathrm{I}$ as low as $0.001 \mathrm{~A}$ can be clearly detected by a step change in the output voltage. Hence, the resolution is much lower than $0.001 \mathrm{~A}$. Moreover, the invariance of voltage with time indicates the good stability and the low effect of hysteresis in the sensor's accuracy.

\subsection{Comparison to the literature}

\subsubsection{Magnetoelectric current sensors}

In order to compare our sensor with those reported in the literature, we summarized the main characteristics of ME current sensors in Table 2. First, it appears that the sensor presented in this study is the only one composed of materials without rare-earth elements. Then, the sensitivity of the sensor seems to be slightly lower than for the others, even though in-plane series connection structure was employed. This is due to the one-turn coil used to produce the magnetic excitation field, which reduces the sensitivity of the sensor. However, this coil permits 


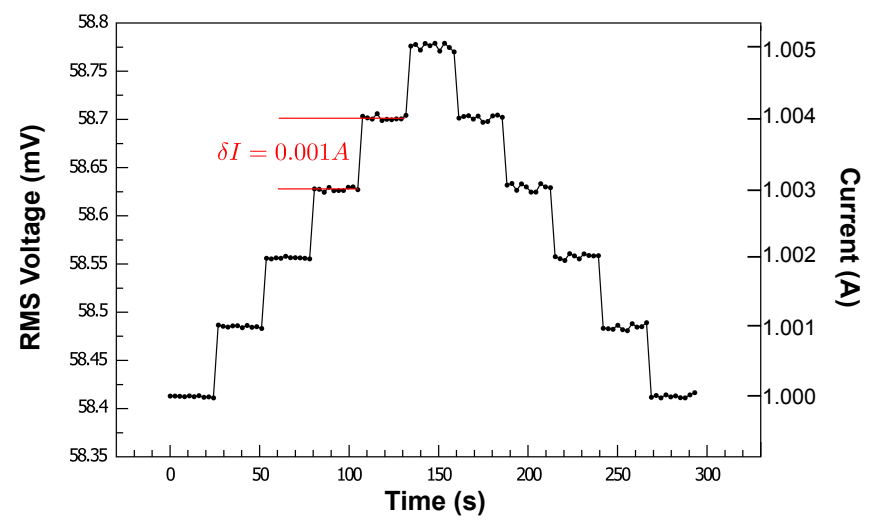

Fig. $15 \mathrm{ME}$ voltage (RMS) measured by the sensor when driving by a $1 \mathrm{~A}$ pk sinusoidal AC current at $5 \mathrm{kHz}$ as a function of time and small current step changes $(\delta \mathrm{I}=0.001 \mathrm{~A}$.)

Table 2 Performance comparison of magnetoelectric current sensors regarding the materials used.

\begin{tabular}{lllllll}
\hline Materials & $\begin{array}{l}\text { Sensitivity } \\
(\mathrm{V} / \mathrm{A})\end{array}$ & $\begin{array}{l}\text { Frequency } \\
(\mathrm{Hz})\end{array}$ & $\begin{array}{l}\text { Linearity } \\
(\mathrm{A})\end{array}$ & $\begin{array}{l}\text { Resolution } \\
(\mathrm{mA})\end{array}$ & Disturbance & Ref. \\
\hline TFD*/PZN-PT & 0.5 & $0.5-10^{5}$ & $\left(10^{-7}\right)-7$ & - & low & {$[6]$} \\
TFD/PZT & 1.03 & $20-5.10^{3}$ & $0.015-2.1$ & - & medium & {$[11]$} \\
TFD/PZT & 0.013 & $1-3.10^{4}$ & $0.015-2.1$ & - & low & {$[7]$} \\
SmF**/PZT & 0.15 & $1-3.10^{4}$ & $0.01-150$ & 10 & possibly high & {$[9]$} \\
NCZF/PZT & 0.09 & $10-3.10^{4}$ & $0.001-30$ & 1 & low & This Study \\
\hline
\end{tabular}

*TFD: Terfenol-D, ${ }^{* *} \mathrm{SmF}=$ Samfenol

to decrease the disturbances induced on the measured signal, because of the low impedance added. It also allows to increase the linearity range for high currents. Additionally, the NCZF/PZT/NCZF magnetoelectric sensor exhibits the highest resolution reported so far. The performances of our rare-earth free magnetoelectric current sensor are hence comparable with the ones obtained with ME composite based on Terfenol-D or Samfenol.

\subsubsection{Commercial current sensors}

Table 3 shows the comparative characteristics of commercial AC current sensing technology. It exists different measurement principle for current sensing and we have chosen to compare our sensor with two technologies widely used: the Hall effect sensor and the inductive sensor. The Hall effect sensors are magnetic field sensors which uses a magnetic flux concentrator while inductive sensor are based on current transformer. Here, we compare our prototype with Hall effect sensors from Asahi Kasei Microdevices (HG-106C) and Infineon Technology (KSY14), and the inductive sensor is from Thai Lin (TL59-A1X-0750). These sensors were chosen because they can be used in a context similar to our sensor.

The current sensor based on Hall effect has a high current capability and a wide frequency range for a very small size, which makes it a sensor suitable for various 
Table 3 Performance comparison of commercial current sensing technology.

\begin{tabular}{llllll}
\hline Sensors & $\begin{array}{l}\text { Measuring } \\
\text { Principle }\end{array}$ & $\begin{array}{l}\text { Frequency } \\
(\mathrm{Hz})\end{array}$ & $\begin{array}{l}\text { Max. } \\
\text { Current }(\mathrm{A})\end{array}$ & $\begin{array}{l}\text { Sensitivity } \\
(\mathrm{V} / \mathrm{mT})\end{array}$ & $\begin{array}{l}\text { Sensitivity } \\
(\mathrm{V} / \mathrm{A})\end{array}$ \\
\hline HG-106C & Hall effect & - & - & 0.0015 & - \\
KSY14 Infineon & Hall effect & $\mathrm{DC}-10^{6}$ & - & 0.0016 & - \\
TL59-A1X-0750 & Transformer & $10^{3}-2.5 .10^{4}$ & 25 & - & 0.067 \\
This study & ME effect & $10-3.10^{4}$ & 30 & 0.78 & 0.09 \\
\hline
\end{tabular}

applications. However, it has the drawback to be expensive and to have a low sensitivity which can only be enhanced by a high-quality signal conditioning electronics. It also requires a biasing current, increasing its power consumption, and a magnetic flux concentrator which enhances the electromagnetic interferences. The inductive sensor shows similar characteristics to our ME sensor but with a lower sensitivity. Here, the advantage of the ME sensor is higher sensitivity, lower cost and simple electronic interface, which makes it a promising technology for current measurements.

\section{Conclusion}

We have fabricated a current sensor based on a ME trilayer composite using an in-plane series connected structure. The design developed here permits to obtain high performances in very compact volume with limited disturbances added to the measured signal. However, the study of our current sensor has shown two main limits: its low cut-off frequency due to the output capacity of the piezoelectric layer, and the oscillations of the ME voltage response after a rising edge of the current, owing to the electro-mechanical resonance. The first issue can be addressed by using a high input impedance buffer, hence reducing the low cut-off frequency to $10 \mathrm{~Hz}$ and less. Regarding the second point, we showed that these oscillations have limited impacts on the ME voltage since the SNR is about $40 \mathrm{~dB}$. Moreover, the rising edge of the current in the field of power electronics is rare, owing to the inductive behaviors of the electronic circuits resulting in triangle shape waveform. We have put in evidence how to properly characterize the sensor and how to optimize the coil's dimension in order to tune the relation sensitivity/disturbance, making a sensor with properties comparable with the ones composed of rareearth elements. In the future, a lead free sensor could also be designed, because promising materials have been proposed lately to replace PZT in magnetoelectric structures $[36,37]$.

\section{References}

1. E. Du Trémolet de Lacheisserie, Magnetostriction, Theory and Applications of Magnetoelasticity (Boca Raton, FL, USA:CRC Press, 1993)

2. C. Fang, J. Jiao, J. Ma, D. Lin, H. Xu, X. Zhao, H. Luo, Significant reduction of equivalent magnetic noise by in-plane series connection in magnetoelectric Metglas/Mn-doped $\mathrm{Pb}(\mathrm{Mg} 1 / 3$ Nb2/3)O3-PbTiO3 laminate composites, J. Phys. D: Appl. Phys. 48(46), $465002(2015)$ 
3. D. Burdin, D. Chashin, N. Ekonomov, L. Fetisov, Y. Fetisov, M. Shamonin, DC magnetic field sensing based on the nonlinear magnetoelectric effect in magnetic heterostructures, J. Phys. D: Appl. Phys. 49(37), 375002 (2016)

4. S. Reis, M. Silva, N. Castro, V. Correia, P. Martins, A. Lasheras, J. Gutierrez, J. Barandiaran, J. Rocha, S. Lanceros-Mendez, Characterization of Metglas/poly(vinylidene fluoride)/Metglas magnetoelectric laminates for $\mathrm{AC} / \mathrm{DC}$ magnetic sensor applications, Materials \& Design 92, 906 (2016)

5. Z. Chu, H. Shi, M. Pourhosseiniasl, J. Wu, W. Shi, X. Gao, X. Yuan, S. Dong, A magnetoelectric flux gate - New approach for weak DC magnetic field detection, Scientific Reports $\mathbf{7}(1), 8592(2017)$

6. S. Dong, J.G. Bai, J. Zhai, J.F. Li, G.Q. Lu, D. Viehland, S. Zhang, T.R. Shrout, Circumferential-mode, quasi-ring-type, magnetoelectric laminate composite-a highly sensitive electric current andor vortex magnetic field sensor, Appl. Phys. Lett. 86(18), 182506 (2005)

7. C.M. Leung, S.W. Or, S. Zhang, S.L. Ho, Ring-type electric current sensor based on ringshaped magnetoelectric laminate of epoxy-bonded Tb0.3Dy0.7Fe1.92 short-fiber/NdFeB magnet magnetostrictive composite and $\mathrm{Pb}(\mathrm{Zr}, \mathrm{Ti}) \mathrm{O} 3$ piezoelectric ceramic, J. Appl. Phys. 107(9), 09D918 (2010)

8. J. Zhang, P. Li, Y. Wen, W. He, A. Yang, C. Lu, J. Qiu, J. Wen, J. Yang, Y. Zhu, M. Yu, High-resolution current sensor utilizing nanocrystalline alloy and magnetoelectric laminate composite, Rev. Sci. Instrum. 83(11), 115001 (2012)

9. J. Zhang, P. Li, Y. Wen, W. He, A. Yang, C. Lu, Packaged current-sensing device with selfbiased magnetoelectric laminate for low-frequency weak-current detection, Smart Mater. Struct. 23(9), 095028 (2014)

10. C. Lu, P. Li, Y. Wen, A. Yang, C. Yang, D. Wang, W. He, J. Zhang, Zero-biased magnetoelectric composite Fe73.5Cu1Nb3Si13.5B9/Ni/Pb(Zr1x,Tix)O3 for current sensing, J. Alloys. Comp. 589, 498 (2014)

11. X. Yu, G. Lou, H. Chen, C. Wen, S. Lu, A slice-type magnetoelectric laminated current sensor, IEEE Sens. J. 15(10), 5839 (2015)

12. V. Loyau, V. Morin, G. Chaplier, M. LoBue, F. Mazaleyrat, Magnetoelectric effect in layered ferrite/PZT composites. Study of the demagnetizing effect on the magnetoelectric behavior, J. Appl. Phys. 117(18), 184102 (2015)

13. V. Loyau, V. Morin, J. Fortineau, M. LoBue, F. Mazaleyrat, A method to decrease the harmonic distortion in Mn-Zn ferrite/PZT and Ni-Zn ferrite/PZT layered composite rings exhibiting high magnetoelectric effects, J. Appl. Phys. 118(15), 154101 (2015)

14. W. He, J. Zhang, C. Qu, J. Wu, J. Peng, A passive electric current sensor based on ferromagnetic invariant elastic alloy, piezoelectric ceramic, and permalloy yoke, IEEE Trans. Magn. 52(7), 1 (2016)

15. M. Bichurin, R. Petrov, V. Leontiev, G. Semenov, O. Sokolov, Magnetoelectric current sensors, Sensors 17(6) (2017)

16. Y. Guo, T. Wang, D. Shi, P. Xiao, Q. Zheng, C. Xu, K.H. Lam, D. Lin, Strong piezoelectricity and multiferroicity in $\mathrm{BiFeO} 3-\mathrm{BaTiO} 3-\mathrm{NdCoO} 3$ lead-free piezoelectric ceramics with high curie temperature for current sensing application, J. Mater. Sci. Mater. Electron. $\mathbf{2 8}(7), 5531(2017)$

17. C.M. Leung, X. Zhuang, D. Friedrichs, J. Li, R.W. Erickson, V. Laletin, M. Popov, G. Srinivasan, D. Viehland, Highly efficient solid state magnetoelectric gyrators, Appl. Phys. Lett. 111(12), 122904 (2017)

18. C.E. Ciomaga, O.G. Avadanei, I. Dumitru, M. Airimioaei, S. Tascu, F. Tufescu, L. Mitoseriu, Engineering magnetoelectric composites towards application as tunable microwave filters, J. Phys. D: Appl. Phys. 49(12), 125002 (2016)

19. Y. Han, L. Li, F. Wang, Y. Yuan, Y. Miao, J. Zhao, K. Zhang, Electric-field switch of magnetization in BaTiO3-Na0.5Bi0.5TiO3-NiFe2O4 composite, J. Mater. Sci. Mater. Electron. 26(11), 8261 (2015)

20. Y. Han, F. Wang, K. Zhang, A novel magnetoelectric memory cell based on bilayer ferroelectric films of (1-x)[Ba(Zr0.2Ti0.8)O3]-x(Ba0.7Ca0.3TiO3), J. Mater. Sci. Mater. Electron. 27(7), 7374 (2016)

21. W. Kleemann, Multiferroic and magnetoelectric nanocomposites for data processing, J. Phys. D: Appl. Phys. 50(22), 223001 (2017)

22. X. Guo, B. Cui, C. Guan, D. Li, K. Wu, J. Yun, T. Wang, Y. Peng, Y. Zuo, L. Xi, Electric field modulation of reversible and non-volatile magnetic anisotropy transition in (011) $0.7[\mathrm{~Pb}(\mathrm{Mg} 1 / 3 \mathrm{Nb} 2 / 3) \mathrm{O} 3]-0.3[\mathrm{PbTi0} 3 \mathrm{O} 3$ ]/FeSi heterostructures, J. Phys. D: Appl. Phys. 50(33), 335001 (2017) 
23. Z. Chen, C. Gao, Y. Wei, P. Zhang, Y. Wang, C. Zhang, Z. Ma, Large non-volatile tuning of magnetism mediated by electric field in $\mathrm{Fe}-\mathrm{Al} / \mathrm{Pb}(\mathrm{Mg} 1 / 3 \mathrm{Nb} 2 / 3) \mathrm{O} 3-\mathrm{PbTiO} 3$ heterostructure, J. Phys. D: Appl. Phys. 50(23), 235005 (2017)

24. Y. Zhou, S. Priya, in Composite Magnetoelectrics, Woodhead Publishing Series in Electronic and Optical Materials (Woodhead Publishing, 2015), p. 209

25. D. Sander, S.O. Valenzuela, D. Makarov, C.H. Marrows, E.E. Fullerton, P. Fischer, J. McCord, P. Vavassori, S. Mangin, P. Pirro, B. Hillebrands, A.D. Kent, T. Jungwirth, O. Gutfleisch, C.G. Kim, A. Berger, The 2017 Magnetism Roadmap, J. Phys. D: Appl. Phys. $\mathbf{5 0}(36), 363001(2017)$

26. V. Loyau, A. Aubert, M. LoBue, F. Mazaleyrat, Analytical modeling of demagnetizing effect in magnetoelectric ferrite/PZT/ferrite trilayers taking into account a mechanical coupling, J. Magn. Magn. Mater. 426, 530 (2017)

27. J. Karki, Signal conditioning piezoelectric sensors. Tech. rep., Texas Instruments (2000)

28. A. Aubert, V. Loyau, F. Mazaleyrat, M. LoBue, Uniaxial anisotropy and enhanced magnetostriction of $\mathrm{CoFe} 2 \mathrm{O} 4$ induced by reaction under uniaxial pressure with SPS, J. Eur. Ceram. Soc. 37(9), 3101 (2017)

29. Y. Liu, G. Xu, H. Lv, C. Huang, Y. Chen, Y. Bie, J. Zhang, T. Liu, N. Hu, G. Deng, L. Pei, J. Shi, R. Xiong, Magnetoelectric ceramic composites prepared by spark plasma sintering with notably enhanced magnetoelectric effect, J. Mater. Sci. Mater. Electron. 28(4), 3746 (2017)

30. M.I. Bichurin, D.A. Filippov, V.M. Petrov, V.M. Laletsin, N. Paddubnaya, G. Srinivasan, Resonance magnetoelectric effects in layered magnetostrictive-piezoelectric composites, Physical Review B 68, 132408 (2003)

31. A. Aubert, V. Loyau, F. Mazaleyrat, M. LoBue, Enhancement of the magnetoelectric effect in multiferroic $\mathrm{CoFe} 2 \mathrm{O} 4 / \mathrm{PZT}$ bilayer by induced uniaxial magnetic anisotropy, IEEE Trans. Magn. 53(11), 1 (2017)

32. H. Xu, Y. Pei, D. Fang, P. Wang, Nonlinear harmonic distortion effect in magnetoelectric laminate composites, Appl. Phys. Lett. 105(1), 012904 (2014)

33. D. Burdin, D. Chashin, N. Ekonomov, L. Fetisov, Y. Fetisov, G. Sreenivasulu, G. Srinivasan, Nonlinear magneto-electric effects in ferromagnetic-piezoelectric composites, J. Magn. Magn. Mater. 358-359, 98 (2014)

34. Y. Shi, Y.W. Gao, Theoretical study on nonlinear magnetoelectric effect and harmonic distortion behavior in laminated composite, J. Alloys. Comp. 646, 351 (2015)

35. D. Burdin, D. Chashin, N. Ekonomov, Y. Fetisov, Static deformation of a ferromagnet in alternating magnetic field, J. Magn. Magn. Mater. 406, 217 (2016)

36. X. Yan, B. Peng, J. Zou, X. Zeng, Evolution of phase structure and enhancement of piezoelectric properties in (K0.5Na0.5)0.94Li0.06NbO3-SrZrO3 lead-free ceramics, J. Mater. Sci. Mater. Electron. 28(4), 3581 (2017)

37. H. Zhang, D.y. Zheng, S.m. Hu, C. Cheng, G.g. Peng, J. Zhang, L.l. Li, Structural and electrical properties of $\mathrm{Bi} 1 / 2 \mathrm{Na} 1 / 2 \mathrm{TiO} 3-\mathrm{BaTiO} 3-\mathrm{Sr} 3 \mathrm{CuNb} 2 \mathrm{O} 9$ lead-free piezoelectric ceramics, J. Mater. Sci. Mater. Electron. 28(1), 67 (2017) 